\title{
Robotic Pancreaticoduodenectomy: Single-Surgeon Initial Experience
}

\author{
Mingjun Wang ${ }^{1} \cdot$ Yunqiang $\mathrm{Cai}^{1} \cdot{\text { Yongbin } \mathrm{Li}^{1} \cdot \operatorname{Bing} \text { Peng }}^{1}$
}

Received: 31 July 2016 / Accepted: 7 October 2016 / Published online: 21 October 2016

(C) Association of Surgeons of India 2016

\begin{abstract}
Minimally invasive surgery has gained increasing acceptance over the last few years, which has expanded to pancreaticoduodenectomy. Laparoscopic pancreaticoduodenectomy has been determined to be a feasible, safe, and effective procedure in an experienced surgeon's hands, but the adaptations to the clumsy instruments are needed. The improved dexterity of the Da Vinci robotic system provides a good opportunity to perform this challenging procedure in the minimally invasive context. The aim of this study was to share our preliminary experience of totally robotic pancreaticoduodenectomy. From April 2015 to August 2015 , four patients were selected to undergo totally robotic pancreaticoduodenectomy in the Department of Pancreatic Surgery, West China Hospital, Sichuan University, China. The demographic characteristics, perioperative details, and pathological results were retrospectively reviewed. One female and two male patients underwent totally robotic pancreaticoduodenectomy, while another male patient underwent robotic total pancreatectomy due to the severe atrophy of pancreatic body and tail. The mean age of the four patients was 56.8 years. The average operation time and intraoperative blood loss were $563 \mathrm{~min}$ and $228 \mathrm{~mL}$, respectively. No one needed blood transfusion, conversion to open pancreaticoduodenectomy, or postoperative analgesia. The postoperative courses of these patients were uneventful. The mean postoperative hospital stay was
\end{abstract}

Bing Peng

wmjjmw01pb@126.com

1 Department of Pancreatic Surgery, West China Hospital, Sichuan University, No. 37 Guoxue Alley, Chengdu, Sichuan 610041, China
10 days. No one required to be readmitted, and there was no death within 30 days following the surgery. Final pathologic examinations revealed one malignant pancreatic ductal adenocarcinoma, and three benign lesions. Based on this initial study, robotic pancreaticoduodenectomy is safe and feasible, with acceptable oncological outcomes for highly selected patients in experienced surgeons' hands. However, concerns such as long-term outcomes, cost-effectiveness analysis, and learning curve analysis should be fully demonstrated before the popularization of this challenging procedure.

Keywords Da Vinci · Pancreaticoduodenectomy · Minimally invasive surgery $\cdot$ Total pancreatectomy

\section{Introduction}

Pancreaticoduodenectomy (PD) is one of the most complicated abdominal surgical procedures, including dissection of the duodenum and pancreas, transection of the pancreas, and reconstruction of the gastrointestinal tract.

Current breakthroughs in technological innovation and surgical strategies have made it practical to perform PD in a minimally invasive context. Two decades after the first description of laparoscopic pancreaticoduodenectomy (LPD) [1], an increasing number of studies have demonstrated the safety and feasibility of LPD, and emphasized its advantages in terms of earlier oral intake, fewer blood loss, shorter postoperative hospital stay, and fewer postoperative complications over its open counterpart [2-5]. Despite these promising initial results for patients, surgeons have to adapt to the clumsy instruments when performing LPD. 
Da Vinci robotic system (dVss), the biggest star in the field of the minimally invasive surgery, can provide many technical advantages over laparoscopic surgery including highdefinition 3D views, enhanced suturing ability, and more degrees of freedom of movement with fully wristed instruments.

Previously, we shared our initial experience of LPD in 31 cases [4], and herein, we reported 4 cases of totally robotic pancreaticoduodenectomies (RPD) in order to explore the feasibility, safety, as well as the acceptable oncological outcomes of this procedure.

\section{Materials and Methods}

From April 2015 to August 2015, four patients were selected to undergo pancreaticoduodenectomy with the Da Vinci robotic system in the Department of Pancreatic Surgery, West China Hospital, Sichuan University, China. The selection criteria for RPD included (1) body mass index (BMI) $<28.0 \mathrm{~kg} / \mathrm{m}^{2}$; (2) ampullary tumors, duodenal tumors restricted to the second part of the duodenum, lower common bile duct tumors, and pancreatic tumors at the pancreatic head; (3) chronic mass pancreatitis at the pancreatic head with no response to conservative treatments; and (4) carefully confirmed resectability of the tumors based on preoperative radiology by a professional radiologist and experienced surgeons [4]. Patients with potential vascular involvement, with a history of previous upper abdominal surgery, with tumors extending to the uncinate, and with severe cardiorespiratory comorbidities were excluded for RPD.

All patients were thoroughly informed about the procedure, the risks, and advantages of RPD, as well as the potential for conversion to laparotomy. Informed consent forms were obtained from all patients in our study, which was approved by the Ethics Committee of Sichuan University.

Data were prospectively accrued to a database, and the clinicopathological data were retrospectively reviewed from the medical record, including demographic characteristics (age, sex, and BMI), preoperative information (laboratory test outcomes, American Society of Anesthesiology (ASA) classification, initial symptoms, and comorbidities), operative details (operation time, blood loss, blood transfusion, and conversion rate), final pathologic findings and postoperative courses (intensive care unit (ICU)) stay, analgesia, recovery details, postoperative hospital stay, complications, and so on).

\section{Operation Procedures}

All the procedures were performed by a single team with an experienced laparoscopic surgeon and an assistant surgeon. Patients were placed in the supine position with legs apart, with the table positioned at $20^{\circ}$ in the reverse Trendelenburg position and slightly tilted to the left side. The tower of the
$\mathrm{dVss}$ is positioned at the head of the patient. The surgeon controls the robot from the dVss console, while the assistant surgeon stands between the patient's legs. With the patients under general anesthesia, a total of five trocars were placed following a concave and arcuated line (Fig. 1a). The 10-mm camera port is placed approximately $2-3 \mathrm{~cm}$ to the right of the umbilicus to improve visualization of the lateral border of the portal vein. The first robotic 8-mm port (R1) is placed approximately 3 $4 \mathrm{~cm}$ cephalad to the camera port lateral to the mid-clavicular lines. An assistant port is placed approximately $4-5 \mathrm{~cm}$ caudad to the camera port along the transverse umbilical lines. The third robotic $8-\mathrm{mm}$ trocar (R3) is placed in the left anterior axillary line $4-5 \mathrm{~cm}$ under the costal line, and the second robotic 8-mm trocar (R2) is placed between the assistant port and R3. The following surgical processes were similar to those of LPD, which was described previously [4]. In detail, the procedure began with a full exploration of the abdominal cavity to reconfirm that the patients did not have metastases or abdominal dissemination. The gastrocolic ligament was dissected below the gastroepiploic vessels using a ultrasonic dissector (Harmonic Scalpel, Ethicon Endo-Surgery, Cincinnati, OH), and then the left and right colonic flexures were also dissected. The right gastroepiploic vessels were identified clipped with hemolock clips and then transected using an ultrasonic dissector. The superior mesenteric vein (SMV) was identified by following the Henle's trunk at the inferior border of the pancreas. At the inferior border of pancreas, a tunnel was carefully created between the pancreatic neck and the SMV or portal vein. A colored tape was passed through the tunnel. The pylorus was identified by following the prepyloric vein, and after the right gastric artery was identified and ligated with hemolock clips, the distal stomach was transected $2 \sim 3 \mathrm{~cm}$ from the pylorus using an endoscopic linear stapler. The Kocher maneuver was performed up to the anterior portion of the aorta. The third portion of the duodenum was completely mobilized from the mesocolon. The ligament of Treitz was dissected from right to left, and the jejunum was transected $15 \sim 20 \mathrm{~cm}$ distal from the Treitz ligament using a linear stapler. The free end of the jejunum was then passed through the root of the mesentery to the supramesocolic compartment. A cholecystectomy was performed, and the hepatoduodenal ligament was skeletonized. The bile duct was transected at the level of the common hepatic duct. The gastroduodenal artery was identified, double ligated with hemolock clips, and transected. The pancreatic neck was transected with ultrasound shears from the inferior border to the superior border of the pancreas, and the pancreatic duct was transected with scissors after being identified. The uncinate process was dissected along the adventitia of the superior mesenteric vessels using Ligasure (Covidien, Mansfield, MA), with the large tributary vessels ligated or clipped. When necessary, standard lymphadenectomy of lymph node stations, including 5, 6, 8a, 12b1, 12b2,12c, 13a, 13b, 14a, 14b, 17a, and 17b, was performed. The specimen was placed in a retrieval bag and then 
Fig. 1 a-d Trocar position and digestive tract reconstruction. a Trocar position. $R l$ the first robotic trocar, $R 2$ the second robotic trocar, $R 3$ the third robotic trocar, $A P$ assistant port, $O P$ optical port. $\mathbf{b}$

Pancreatojejunostomy. c Hepaticojejunostomy. d Gastrojejunostomy

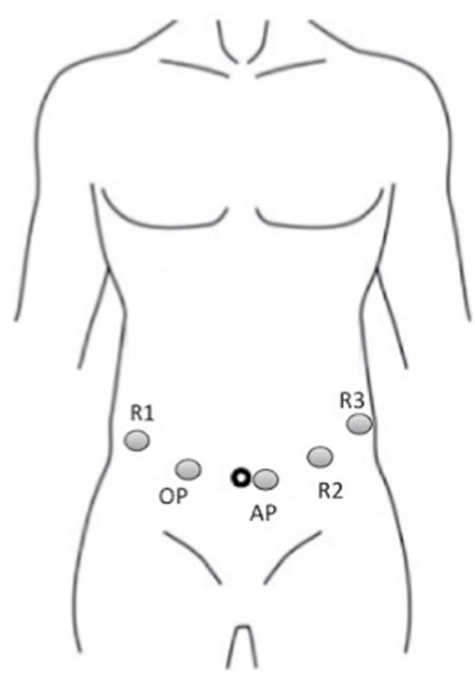

a

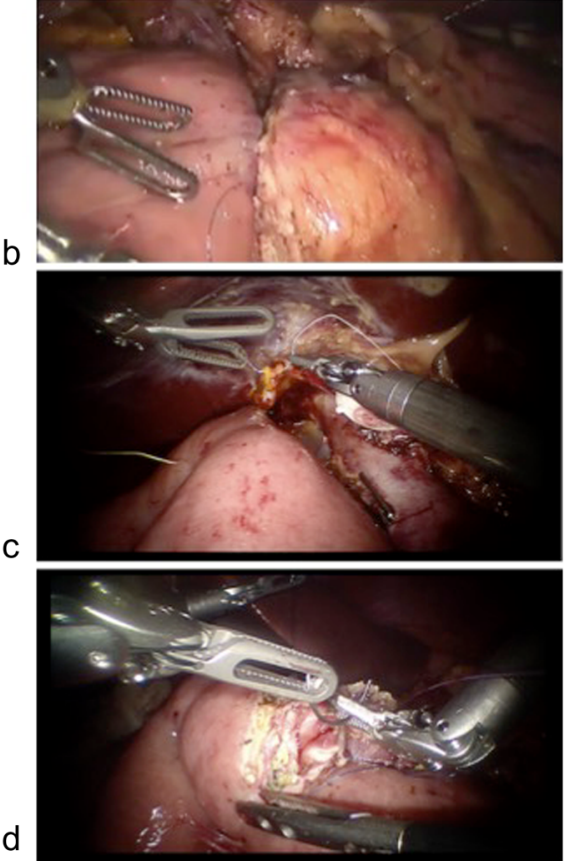

moved through the umbilical site, which was extended approximately $5 \mathrm{~cm}$. The cut edge of the bile duct and pancreatic stump were sent for frozen pathological examination. After the margin status was determined, the incision of the extraction site was closed and the pneumoperitoneum was reestablished again. The reconstruction was performed in the following order: pancreatojejunostomy (Fig. 1b), hepaticojejunostomy (Fig. 1c), and gastrojejunostomy (Fig. 1d). A two-layer duct-to-mucosa anastomosis was formed for the pancreatojejunostomy. Approximately $10 \mathrm{~cm}$ distal to the two-layer duct-to-mucosa anastomosis, an end-to-side hepaticojejunostomy was performed. Antecolic gastrojejunostomy was performed $45 \mathrm{~cm}$ downstream from the hepaticojejunostomy. Operative drains were routinely used as follows: one in Morrison's pouch, one near the hepaticojejunostomy, and one superior and inferior to the pancreatojejunostomy. Finally, all trocars were removed, and the trocar sites were closed with absorbable sutures.

Particularly, in one patient (Case 4), the operation began as in RPD, and then was converted to robotic total pancreatectomy due to the intraoperative findings. For this patient, the distal pancreas was dissected off the splenic vessels from the pancreatic body to the splenic hilum, with the splenic vessels preserved. Once the distal pancreas is fully mobilized, the next resection processes resembled RPD. After the specimen was retrieved and moved through the umbilical site, which was extended approximately $5 \mathrm{~cm}$, the hepaticojejunostomy was performed in the end-to-side manner followed by the antecolic gastrojejunostomy $40-45 \mathrm{~cm}$ downstream from the hepaticojejunostomy. Three closed suction drains were used for this case, with one in Morrison's pouch and two in the bed of distal pancreas.

\section{Results}

Robotic pancreaticoduodenectomy was successfully carried out for three out of the four patients, while the remaining single case needed to be converted to robotic total pancreatectomy because of the severe atrophy of the pancreatic body and tail. The demographic characteristics and preoperative information are listed in Table 1. The mean age of the four patients (three males and one female) was 56.8 years (range 45 to 68 years). The mean BMI was $20.3 \mathrm{~kg} / \mathrm{m}^{2}$ (range 17.6 to $22.0 \mathrm{~kg} / \mathrm{m}^{2}$ ), and all patients were classified as ASA II. Initial symptoms included jaundice, epigastric pain and distension, pruritus, and hyperglycemia with necrolytic migratory erythema, while preoperative comorbidities included renal calculus, hypertension, type II diabetes mellitus, gallstones, and urinary tract infection. Liver function was normal in Cases 2 and 4, while increased total bilirubin and transaminases were detected in Cases 1 and 3. The serum levels of carcinoembryonic antigen (CEA) in the other three cases were normal except the slightly increased level in Case 4, while the increased levels of carbohydrate antigen 19-9 (CA 19-9) were detected in Cases 1,2, and 3 except the normal level in Case 4.

The intraoperative and postoperative details are given in Table 2. The average operation time was $563 \mathrm{~min}$ (range 480 to $600 \mathrm{~min}$ ). The mean blood loss was $228 \mathrm{~mL}$ (range 150 to $270 \mathrm{~mL}$ ). Conversion to open pancreaticoduodenectomy (OPD) did not occur in all patients. All patients experienced 1-day ICU stay. Postoperative analgesia was not required. The mean time to being able to stand and walk without assistance, the first passage of flatus, and the first oral intake were 3.0, 3.0 , and 5.8 days, respectively. Postoperative hospital 
Table 1 Demographic characteristics and preoperative information

\begin{tabular}{|c|c|c|c|c|}
\hline Parameters & Case 1 & Case 2 & Case 3 & Case 4 \\
\hline Age (years) & 68 & 65 & 45 & 49 \\
\hline Sex & Male & Female & Male & Male \\
\hline BMI $\left(\mathrm{kg} / \mathrm{m}^{2}\right)$ & 20.1 & 21.6 & 22.0 & 17.6 \\
\hline ASA score & II & II & II & II \\
\hline Symptoms & Jaundice & $\begin{array}{l}\text { Epigastric pain } \\
\text { and distension }\end{array}$ & $\begin{array}{l}\text { Jaundice, pruritus, } \\
\text { and epigastric pain }\end{array}$ & $\begin{array}{l}\text { Hyperglycemia for } 8 \text { years with } \\
\text { necrolytic migratory erythema }\end{array}$ \\
\hline Total bilirubin $(\mu \mathrm{mol} / \mathrm{L})$ & 112.0 & 9.8 & 127.4 & 2.9 \\
\hline ALT (IU/L) & 141 & 17 & 66 & 41 \\
\hline AST (IU/L) & 152 & 19 & 42 & 19 \\
\hline Albumin $(\mathrm{g} / \mathrm{L})$ & 34.4 & 45.8 & 43.6 & 36.1 \\
\hline CEA (ng/ml) & 2.76 & 1.99 & 3.03 & 6.22 \\
\hline CA $19-9(\mathrm{u} / \mathrm{ml})$ & 83.21 & 41.71 & 32.25 & 21.66 \\
\hline Comorbidities & Renal calculus & $\begin{array}{l}\text { Hypertension and type II } \\
\text { diabetes mellitus }\end{array}$ & $\begin{array}{l}\text { Gallstones and type II } \\
\text { diabetes mellitus }\end{array}$ & Urinary tract infection \\
\hline
\end{tabular}

$B M I$ body mass index, ASA American Society of Anesthesiology, ALT alanine aminotransferase, $A S T$ aspartate aminotransferase, $C E A$ carcinoembryonic antigen, $C A$ 19-9 carbohydrate antigen 19-9

stay ranged from 8 to 12 days, with a mean of 10 days. The mass sizes were $2.0,4.5,3.0$, and $3.0 \mathrm{~cm}$, respectively. The final pathological results revealed one duodenal papilla inflammatory mass, one pancreatic serous cystic neoplasm, one pancreatic ductal adenocarcinoma, and one glucagonoma at the pancreatic head together with serous cystic neoplasm at the body and tail. Especially, the ampullary tumor could not be excluded in Case 1, although preoperative endoscopic retrograde cholangiopancreatography (ERCP) with biopsy was performed. After the patient and his family members being well informed, RPD was ultimately performed for this patient.

The postoperative courses were uneventful in all patients. None of postoperative complications were detected. No one needed to be readmitted within 30 days after surgery. The 30-day mortality after surgery was zero.

Table 2 Intraoperative and postoperative details

\begin{tabular}{lllll}
\hline Parameters & Case 1 & Case 2 & Case 3 & Case 4 \\
\hline Operation time (min) & 480 & 600 & 600 & 570 \\
Blood loss (mL) & 150 & 230 & 270 & 260 \\
Blood transfusion & No & No & No & No \\
Conversion to OPD & No & No & No & 3.0 \\
Size of the mass (cm) & 2.0 & 4.5 & 3.0 & 1 \\
ICU stay (days) & 1 & 1 & 1 & No \\
Postoperative analgesia & No & No & No & 4 \\
Able to stand and walk without & 3 & 2 & 3 & 3 \\
$\quad$ assistance (days) & 4 & 3 & 2 & 6 \\
Time to first passage of flatus (days) & 7 & 5 & 5 & 8 \\
Time to oral intake (days) & 9 & 12 & 11 & No \\
Postoperative hospital stay (days) & No & No & No & Glucagonoma (NET G2) at \\
Complications & Duodenal papilla & Pancreatic serous & Pancreatic ductal & adenocarcinoma \\
Final pathologic results & inflammatory mass & cystic neoplasm & T3N1M0 (IIB) & cystic neoplasm at the body and tail \\
& & No & No & No
\end{tabular}

OPD open pancreaticoduodenectomy, ICU intensive care unit, T3N1M0 (IIB) TNM staging and clinical staging were based on AJCC/UICC Staging Manual, Seventh Edition, (NET G2) the tumor was classified according to the WHO 2010 classification system for pancreatic endocrine tumors (PETs) 


\section{Discussion}

Pancreaticoduodenectomy has historically been regarded as one of the most complex abdominal surgical operations, with high postoperative morbidities. Since the first laparoscopic operation was introduced in the late 1980s, surgical procedures have been changing from open to minimally invasive manner. Gagner and Pomp first reported LPD in 1994 and demonstrated that LPD was feasible and safe, and could replicate the steps of open operation [1]. Afterwards, enthusiasm to perform LPD decreased, and this procedure was almost performed in experienced surgeons' hands in expert centers due to the fact that the limitations of LPD including reduced freedom of movement, 2D view, reduced precision, and poor ergonomics required a lot of time and effort to develop and maintain such advanced skills.

It is the shortcomings of laparoscopic surgery that promotes the development of robotic surgery. Compared with traditional laparoscopy surgery, the robotic technique can provide more advantages such as precise tissue manipulation, 3D imaging, elimination of surgeon tremor, and the articulation of the robotic arms with almost $540^{\circ}$ of motion [6]. In addition, the surgeon's fatigue may decrease due to the ergonomic benefits [7].

The first large case series of RPD enrolling 60 patients were reported by Giulianotti et al. in 2010, indicating that RPD was a safe and feasible procedure [8]. And next came another large case series of 50 RPDs by Zeh et al. in 2013, obtaining the same conclusion [9]. RPD is a time-consuming procedure, and according to the largest case series of RPDs published to date, the operative time for RPD ranged from 420 to $718 \mathrm{~min}$ [8-15]. In the current study, our data showed a mean operative time of $563 \mathrm{~min}$ (range 480 to $600 \mathrm{~min}$ ), which was in accordance with the reported values. Someone may criticize the increased operative time when comparing with OPD, indicating the longer operative time would do harm to the patients. However, this is partially because of the set-up and docking time, which can be shorten over time with increased experience [16].

Postoperative pancreatic fistula (POPF) is the major concern of PD. The incidences of POPF after OPD and LPD were reported to range from 2.0 to $36.0 \%$, and from 0 to $35.0 \%$, respectively [17-21]. When it came to RPD, the reported incidence ranged from 7.0 to $35.0 \%[8,9,11-13,15]$, and two major systemic reviews published to date indicated there was no significant difference between RPD and OPD in terms of POPF [7, 22]. However, Lai et al. reported a higher POPF rate of RPD in comparison to that of OPD [15]. Excitingly, in the current study, none of the four patients developed POPF, which may result from the rich experience of the surgeon (Bing Peng) who have successfully completed 70 LPDs until September 2015. Overall, with improvement in technique and more experience with laparoscopic reconstructions, the incidence of POPF will decrease.
Another concern is the appropriateness of RPD for patients with malignancy. It remains unclear that whether minimally invasive PD is superior to OPD regarding the amounts of lymph nodes collected and the R0 resection ratio. Several authors have reported acceptable oncological outcomes of RPD. In Lai's study, comparing 20 patients undergoing RPD with 67 patients undergoing OPD, the R0 resection rates and harvested lymph node numbers were not significantly different between the two groups [15]. And except a higher rate of positive margins in OPD, the similar outcome regarding the number of harvested lymph nodes was obtained in Chalikonda's study, in which 30 patients with RPD and 30 patients with OPD were enrolled [12]. Furthermore, in Zhang's meta-analysis, RPD was reported to be associated with lower positive margin rate [22]. In the present study, the final pathologic examination verified a R0 resection in the single patient (Case 3) with malignancy, with 13 harvested lymph nodes. Even so, whether RPD is superior to OPD in terms of oncological outcomes should be determined by longterm survival outcomes; however, long-term data of RPD are scarce until now.

In one of the four cases in this study (Case 4), the intended RPD was converted to robotic total pancreatectomy due to the intraoperative findings (severe atrophy of the pancreatic body and tail). In this case, the spleen was preserved considering the important immunological functions of the spleen, and some complications resulting from splenectomy, such as overwhelming postsplenectomy sepsis, late malignancies, and venous thrombosis [23]. It was the flexible robotic joints that allowed the surgeon to individualize both the splenic artery and vein branches and to selectively ligate them precisely, which contributed to the success of the spleen-preserving robotic total pancreatectomy.

Although the reported outcomes of RPD were promising, the use of robotics in pancreaticoduodenectomy is limited and still in its infancy, making it difficult to clarify long-term benefits to patients. The other disadvantages, if any, of robotic surgery are the lack of haptic feedback and the increased procedure-related costs [24, 25]. However, one should hold the belief that robot does have a role in complicated procedures such as pancreaticoduodenectomy.

Admittedly, this study has some limitations. This is a retrospective preliminary study with only four cases in a single academic center. The results may be questioned because of the high selection criteria. Long-term results were not available because RPD started just 5 months ago in our center. Furthermore, it is very difficult to carry out a prospective study with a large number of patients to compare RPD with OPD or LPD in a single center, therefore the cooperation of multiple centers is required to draw definitive conclusions regarding the role of RPD. 
In summary, based on this initial study, RPD is safe and feasible, with acceptable oncological outcomes for highly selected patients in experienced surgeons' hands.

Acknowledgments This study was funded by the West China Hospital, Sichuan University.

Author Contributions Conceived and designed the experiments: Mingjun Wang. Li.

Performed the experiments: Mingjun Wang, Yunqiang Cai, Yongbin

Analyzed the data: Mingjun Wang.

Wrote the paper: Mingjun Wang.

Final approval of the version to be published: Bing Peng.

\section{Compliance with Ethical Standards}

Conflict of Interest The authors declare that they have no conflict of interest.

\section{References}

1. Gagner M, Pomp A (1994) Laparoscopic pylorus-preserving pancreatoduodenectomy. Surg Endosc 8:408-410

2. Croome KP, Farnell MB, Que FG, Reid-Lombardo K, Truty MJ, Nagorney DM, Kendrick ML (2014) Total laparoscopic pancreaticoduodenectomy for pancreatic ductal adenocarcinoma: oncologic advantages over open approaches? Ann Surg 260:633640

3. Langan RC, Graham JA, Chin AB, Rubinstein AJ, Oza K, Nusbaum JA, Smirniotopoulos J, Kayser R, Jha R, Haddad N, Al-Kawas F, Carroll J, Hanna J, Parker A, Al-Refaie WB, Johnson LB (2014) Laparoscopic-assisted versus open pancreaticoduodenectomy: early favorable physical quality-of-life measures. Surgery 156:379-384

4. Wang M, Zhang H, Wu Z, Zhang Z, Peng B (2015) Laparoscopic pancreaticoduodenectomy: single-surgeon experience. Surg Endosc 29:3783-3794

5. Song KB, Kim SC, Hwang DW, Lee JH, Lee DJ, Lee JW, Park KM, Lee YJ (2015) Matched case-control analysis comparing laparoscopic and open pylorus-preserving pancreaticoduodenectomy in patients with periampullary tumors. Ann Surg 262:146-155

6. Zeh HJ 3rd, Bartlett DL, Moser AJ (2011) Robotic-assisted major pancreatic resection. Adv Surg 45:323-340

7. Strijker M, van Santvoort HC, Besselink MG, van Hillegersberg R, Borel Rinkes IH, Vriens MR, Molenaar IQ (2013) Robot-assisted pancreatic surgery: a systematic review of the literature. HPB (Oxford) 15:1-10

8. Giulianotti PC, Sbrana F, Bianco FM, Elli EF, Shah G, Addeo P, Caravaglios G, Coratti A (2010) Robot-assisted laparoscopic pancreatic surgery: single-surgeon experience. Surg Endosc 24:16461657
9. Zeh HJ, Zureikat AH, Secrest A, Dauoudi M, Bartlett D, Moser AJ (2012) Outcomes after robot-assisted pancreaticoduodenectomy for periampullary lesions. Ann Surg Oncol 19:864-870

10. Narula VK, Mikami DJ, Melvin WS (2010) Robotic and laparoscopic pancreaticoduodenectomy: a hybrid approach. Pancreas 39: 160-164

11. Buchs NC, Addeo P, Bianco FM, Ayloo S, Benedetti E, Giulianotti PC (2011) Robotic versus open pancreaticoduodenectomy: a comparative study at a single institution. World J Surg 35:2739-2746

12. Chalikonda S, Aguilar-Saavedra JR, Walsh RM (2012) Laparoscopic robotic-assisted pancreaticoduodenectomy: a casematched comparison with open resection. Surg Endosc 26:23972402

13. Zhou NX, Chen JZ, Liu Q, Zhang X, Wang Z, Ren S, Chen XF (2011) Outcomes of pancreatoduodenectomy with robotic surgery versus open surgery. Int J Med Robot 7:131-137

14. Boggi U, Signori S, De Lio N, Perrone VG, Vistoli F, Belluomini M, Cappelli C, Amorese G, Mosca F (2013) Feasibility of robotic pancreaticoduodenectomy. Br J Surg 100:917-925

15. Lai EC, Yang GP, Tang CN (2012) Robot-assisted laparoscopic pancreaticoduodenectomy versus open pancreaticoduodenectomy - a comparative study. Int J Surg 10:475-479

16. Iranmanesh $\mathrm{P}$, Morel $\mathrm{P}$, Wagner OJ, Inan I, Pugin F, Hagen ME (2010) Set-up and docking of the da Vinci surgical system: prospective analysis of initial experience. Int J Med Robot 6:57-60

17. Mabrut JY, Fernandez-Cruz L, Azagra JS, Bassi C, Delvaux G, Weerts J, Fabre JM, Boulez J, Baulieux J, Peix JL, Gigot JF, Hepatobiliary, Pancreatic Section of the Royal Belgian Society of S, Belgian Group for Endoscopic S, Club C (2005) Laparoscopic pancreatic resection: results of a multicenter European study of 127 patients. Surgery 137:597-605

18. Gagner M, Pomp A (1997) Laparoscopic pancreatic resection: is it worthwhile? J Gastrointest Surg 1:20-25 discussion 5-6

19. Dulucq JL, Wintringer P, Mahajna A (2006) Laparoscopic pancreaticoduodenectomy for benign and malignant diseases. Surg Endosc 20:1045-1050

20. Bassi C, Dervenis C, Butturini G, Fingerhut A, Yeo C, Izbicki J, Neoptolemos J, Sarr M, Traverso W, Buchler M (2005) Postoperative pancreatic fistula: an international study group (ISGPF) definition. Surgery 138:8-13

21. Bao PQ, Mazirka PO, Watkins KT (2014) Retrospective comparison of robot-assisted minimally invasive versus open pancreaticoduodenectomy for periampullary neoplasms. J Gastrointest Surg 18:682-689

22. Zhang J, Wu WM, You L, Zhao YP (2013) Robotic versus open pancreatectomy: a systematic review and meta-analysis. Ann Surg Oncol 20:1774-1780

23. Beane JD, Pitt HA, Nakeeb A, Schmidt CM, House MG, Zyromski NJ, Howard TJ, Lillemoe KD (2011) Splenic preserving distal pancreatectomy: does vessel preservation matter? J Am Coll Surg 212: 651-657 discussion 7-8

24. Kendrick ML (2012) Laparoscopic and robotic resection for pancreatic cancer. Cancer J 18:571-576

25. Baker EH, Ross SW, Seshadri R, Swan RZ, Iannitti DA, Vrochides D, Martinie JB (2016) Robotic pancreaticoduodenectomy: comparison of complications and cost to the open approach. Int J Med Robot 12:554-560 